\title{
缺陷调控石墨烯复合催化剂在氧还原反应中的作用
}

\author{
蒋丽丽, 徐帅帅, 夏宝凯, 陈胜, 朱俊武 \\ (南京理工大学 软化学与功能材料教育部重点实验室, 南京 210094)
}

摘 要: 电化学氧还原反应(ORR)在能源、催化等领域具有广阔的应用前景, 因此开发性能优异、选择性高的催化剂 对于促进 ORR 发展具有重要意义。ORR 反应按照反应过程可以分为二电子反应过程和四电子反应过程。本研究 以化学修饰石墨烯为原料, 通过调控其表面缺陷并与银-对苯二琨二甲烷(Ag-TCNQ)纳米点复合, 合成了不同缺陷 程度的复合催化剂, 在此基础上比较了 Ag-TCNQ/高缺陷石墨烯和 Ag-TCNQ/低缺陷石墨烯的 ORR 性能。研究结 果显示 $\mathrm{Ag}-\mathrm{TCNQ} /$ 高缺陷石墨烯催化 ORR 的电子转移数为 2.4 , 双氧水产率达 $0.62 \mathrm{mg} / \mathrm{h}$, 法拉第效率为 $64.45 \%$ 。 相比之下, $\mathrm{Ag}-\mathrm{TCNQ} /$ 低缺陷石墨烯参与 $\mathrm{ORR}$ 的电子转移数为 3.7, 氧还原半波电位约为 $0.7 \mathrm{~V}(v s . \mathrm{RHE})$ 。因此, 高 缺陷催化剂促进 ORR 的二电子过程, 而低缺陷的催化剂促进 ORR 的四电子过程。在复合材料中, Ag-TCNQ 纳米 颗粒和石墨烯发挥了各自的结构优势，形成复合效应，共同提高了催化活性。

关 键 词: 石墨烯; 缺陷调控; 银-7,7,8,8-四氰基苯醌二甲烷; 氧还原反应

中图分类号: 0646 文献标志码: A

\section{Defect Engineering of Graphene Hybrid Catalysts for Oxygen Reduction Reactions}

\author{
JIANG Lili, XU Shuaishuai, XIA Baokai, CHEN Sheng, ZHU Junwu
}

(Key Laboratory for Soft Chemistry and Function Materials Ministry of Education, Nanjing University of Science and Science and Technology, Nanjing 210094, China)

\begin{abstract}
Oxygen reduction reaction (ORR) is important for energy and catalytic applications. Therefore, it is significant to develop highly active and selective catalysts to promote ORR. According to the reaction process, ORR can be categorized into two- and four-electrons transfer pathways. In this work, chemically modified graphene sheets with different defects were used as precursors, which were integrated with Ag-7,7,8,8-tetracyanoquinodimethane (Ag-TCNQ) to form hybrid catalysts. The ORR activities of Ag-TCNQ/high defect graphene and Ag-TCNQ/low defect graphene were compared. The results show that the electron transfer number of ORR with Ag-TCNQ/high defect graphene is 2.4. And its corresponding production yield of $\mathrm{H}_{2} \mathrm{O}_{2}$ is $0.62 \mathrm{mg} / \mathrm{h}$, and Faraday efficiency is $64.45 \%$. In comparison, the electron transfer number of ORR with Ag-TCNQ/low defect graphene is 3.7 and the corresponding half-wave potential is about $0.7 \mathrm{~V}$ ( $v s$. RHE). Therefore, high structural defects promote the two-electron process of ORR, while low structural defects facilitate the four-electron process of ORR. In the composites, Ag-TCNQ nanoparticles and graphene have formed a hybrid effect to improve the catalytic activities.
\end{abstract}

Key words: graphene; defect engineering; Ag-7,7,8,8-tetracyanoquinodimethane; oxygen reduction reaction

收稿日期：2021-03-25; 收到修改稿日期：2021-06-22; 网络出版日期：2021-07-12

基金项目: 江苏省自然科学基金(BK20190460); 江苏省双创人才计划

Natural Science Foundation of Jiangsu Province (BK20190460); Jiangsu Innovative/Entrepreneurial Talent Program

作者简介: 蒋丽丽(1994-), 女, 博士研究生. E-mail: 1325406260@qq.com JIANG Lili(1994-), female, PhD candidate. E-mail: 1325406260@qq.com

通信作者: 陈 胜, 教授. E-mail: sheng.chen@njust.edu.cn; 朱俊武, 教授. E-mail: zhujw@njust.edu.cn CHEN Sheng, professor. E-mail: sheng.chen@njust.edu.cn; ZHU Junwu, professor. E-mail: zhujw@njust.edu.cn 
近年来, 利用电化学方法进行能源储存与转换 逐渐成为开发新型清洁能源器件的重要途经 ${ }^{[1-2]}$ 。其 中, 氧还原反应(Oxygen reduction reaction, ORR)按 照反应路径可分为二电子途径和直接四电子途径, 在碱性环境下反应步骤如式(1 3)所示 ${ }^{[3]}$ :

四电子途径:

$$
\mathrm{O}_{2}+2 \mathrm{H}_{2} \mathrm{O}+4 \mathrm{e}^{-} \rightarrow 4 \mathrm{OH}^{-}
$$

二电子途径:

$$
\begin{gathered}
\mathrm{O}_{2}+\mathrm{H}_{2} \mathrm{O}+2 \mathrm{e}^{-} \rightarrow \mathrm{HO}_{2}^{-}+\mathrm{OH}^{-} \\
\mathrm{HO}_{2}^{-}+\mathrm{H}_{2} \mathrm{O}+2 \mathrm{e}^{-} \rightarrow 3 \mathrm{OH}^{-}
\end{gathered}
$$

ORR 的两种反应途径都具有重要的实际应用 价值, 可分别应用于双氧水合成、燃料电池等化工 和能源领域 ${ }^{[4-9]}$ 。例如, 作为一种重要的能源转换装 置 ${ }^{[10]}$, 燃料电池通过电化学过程将化学能转化为电 能 ${ }^{[11]}$, 为了保证燃料电池的高效能源转换, 需要开 发高性能、低成本的阴极 ORR 催化剂, 实现快速的 四电子转移过程。双氧水 $\left(\mathrm{H}_{2} \mathrm{O}_{2}\right.$, 碱性环境下以 $\mathrm{HO}_{2}$ 形式存在)是一种环境友好型氧化剂, 其氧化产物 只有水和氧气，被广泛应用于杀菌消毒、废水处理、 地下水修复等领域 ${ }^{[11-12]}$ 。当前工业合成双氧水的主 要方法为蒽醌法, 这种方法能耗大、成本高、易造 成环境污染 ${ }^{[13]}$ 。开发高效、环保的双氧水生产方法 得到越来越多的重视和研究, ORR 的二电子途径就 是一种极有前景的方法 ${ }^{[14-15]}$ 。综上所述, ORR 作为 一种重要的电化学反应, 其二电子途径和四电子途 径都具有重要的研究价值, 需要开发高活性、高选 择性、高稳定性的催化剂来有效调控 ORR 的反应 过程。

根据电催化反应原理, ORR 涉及的电化学反应 主要发生在固、液、气三相界面, 离子、分子在催 化活性位点上进行反应 ${ }^{[16]}$ 。在设计 ORR 催化剂的 过程中, 可以通过控制催化剂的组成、尺寸、孔隙率 以及界面结构等调控电子转移及物质传输 ${ }^{17-19]}$ 。近年
来, 缺陷调控作为研究电催化剂的新方法引起了广

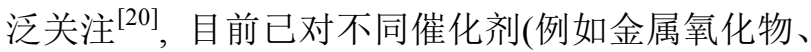
碳化物、过渡金属硫化物等)的缺陷开展了深入研究, 并从理论和实验的角度探索了缺陷与电催化反应过 程之间的构效关系 ${ }^{[21-22]}$ 。研究发现, 缺陷位点通常 表现出独特的电子调控特性, 这对电催化反应尤为 重要。缺陷不仅可以改变原子周围的电子结构, 还 可以作为针点固定其他金属原子, 形成新的协同配 位活性位点，在催化材料应用中十分重要。利用缺陷 的特点设计理想的催化剂具有广阔的研究前景。

石墨烯作为一种新型的二维碳材料, 具有比表 面积大、电子迁移率高、热导率高和机械强度高等 特性, 石墨烯 ${ }^{[23]}$ 及其复合材料 ${ }^{[24-26]}$ 在化学、生物、 医学等多个领域有广泛的应用前景。石墨烯作为碳 材料, 本征催化活性位点较少。本工作合成了不同 缺陷含量的石墨烯, 并与银-7,7,8,8-四㲵基对苯二 琨二甲烷(Ag-TCNQ)进行复合, 制备得到了具有不 同缺陷含量的复合催化剂, 应用于氧还原反应, 并 在此基础上研究缺陷与氧还原反应过程的关系。

\section{1 实验方法}

\section{1 材料的制备(图 1)}

低缺陷石墨烯 称取 $1 \mathrm{~g}$ 石墨粉, 与 $500 \mathrm{~mL} \mathrm{~N}$ 甲基吡咯烷酮(NMP)混合, 水浴超声 $10 \mathrm{~h}$ 后在室温 下静置 $10 \mathrm{~h}$, 然后取上层分散液, 得到低缺陷石墨 烯的 NMP 分散液，标定浓度后保存。

Ag-TCNQ/低缺陷石墨烯 称取 $20 \mathrm{mg}$ 硝酸银 溶解于 $1 \mathrm{~mL}$ 去离子水中, 量取 $19 \mathrm{~mL}$ 低缺陷石墨烯 分散液 $(0.2 \mathrm{mg} / \mathrm{mL})$, 混合均匀后在室温下静置 $4 \mathrm{~h}$ 。 再称取 $20 \mathrm{mg}$ 7,7,8,8-四氰基对苯二琨二甲烷(TCNQ) 配体，溶解于 $10 \mathrm{~mL}$ 乙腈溶剂，与 $\mathrm{Ag} /$ 低缺陷石墨烯

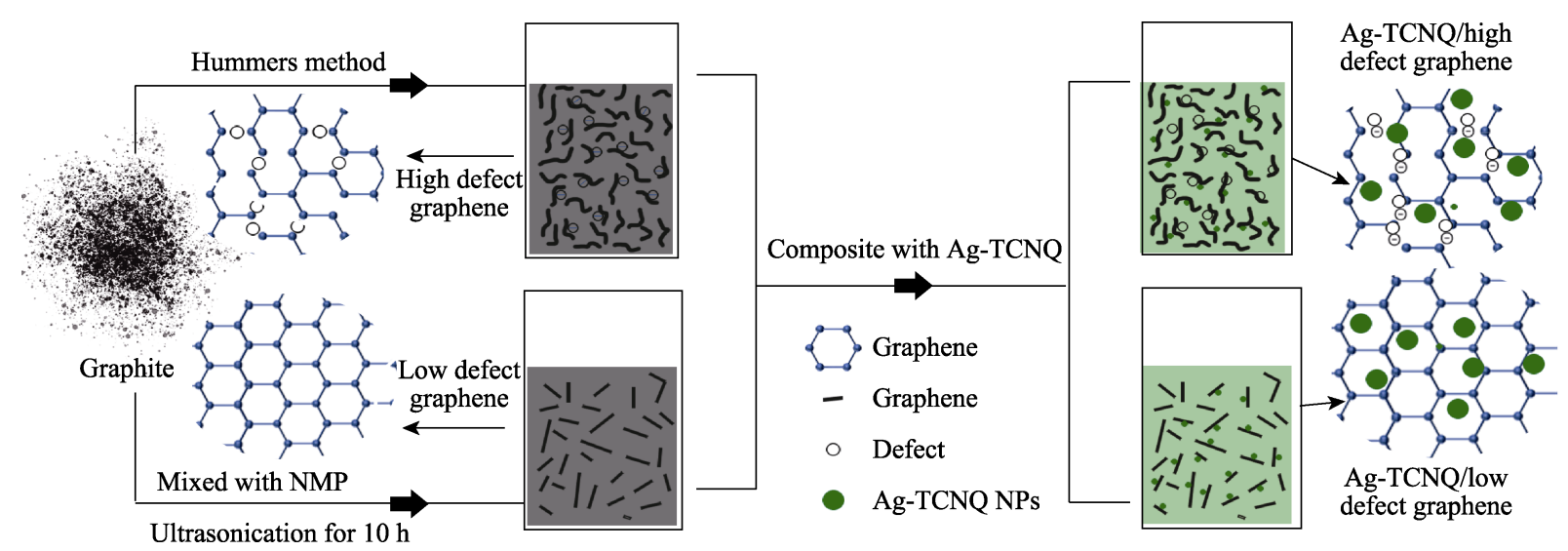

图 1 材料制备过程示意图

Fig. 1 Schematic diagram of material preparation process 
分散液混合后在室温下静置 $2 \mathrm{~h}$ 。最后, 用乙腈、水 离心洗涤后分离产物, 冷冻干燥。

高缺陷石墨烯 采用 Hummers 法[27]制备氧化 石墨，将得到的氧化石墨用去离子水稀释到 $2 \mathrm{mg} / \mathrm{mL}$, 然后水浴超声 $30 \mathrm{~min}$, 得到高缺陷石墨 烯分散液。

Ag-TCNQ/高缺陷石墨烯 首先将 $20 \mathrm{mg}$ 硝酸 银、 $1 \mathrm{~mL}$ 去离子水、19 mL NMP 混合，摚拌溶解混 合均匀后得到硝酸银溶液, 然后与 $1.75 \mathrm{~mL}$ 高缺陷 石墨烯分散液混合后静置 $4 \mathrm{~h}$ 。同时, 取 $20 \mathrm{mg}$ $\mathrm{TCNQ}$ 与 $10 \mathrm{~mL}$ 乙腈配置溶液, 与上述溶液混合静 置反应 $2 \mathrm{~h}$ 。最后，分别用乙腈、水离心洗涤后分离 产物, 冷冻干燥。

\section{2 材料表征}

使用场发射扫描电子显微镜(FESEM，JEOL7800F)、透射电子显微镜(FEI TalosF200x)观察样品 的微观形貌; 使用 X 射线衍射仪(Smart Lab $9 \mathrm{~kW}$, 工作电压为 $40 \mathrm{kV}$, 工作电流为 $40 \mathrm{~mA}, \lambda=0.15406 \mathrm{~nm}$ ) 分析材料的结构; 使用拉曼光谱测试仪(HORIBA Jobin Yvon Aramis, 激发光源波长为 $532 \mathrm{~nm}$ ) 测试样 品的分子结构; 使用紫外-可见分光光度计(Carry-60) 测试紫外-可见光吸收光谱。

\section{3 电化学表征}

旋转环盘电极(RRDE)测试 称取 $5 \mathrm{mg}$ 待测样 品与 $720 \mu \mathrm{L}$ 异丙醇、 $250 \mu \mathrm{L}$ 超纯水、 $30 \mu \mathrm{L}$ 全氟磺 酸型聚合物(Nafion)溶液(质量分数为 $5 \%$ ) 混合, 得 到均匀的待测样品分散液 (浓度为 $5 \mathrm{mg} / \mathrm{mL}$ )。然后 取 $10 \mu \mathrm{L}$ 分散液滴在玻碳电极表面，在室温下干燥 后即得到工作电极。电解液为 $0.1 \mathrm{~mol} / \mathrm{L} \mathrm{KOH}$ 水溶 液, 参比电极为 $\mathrm{Ag} / \mathrm{AgCl}$ 电极, 对电极为铂丝电极。 在氧还原测试前, 向电解液中持续通入高纯氧气 $30 \mathrm{~min}$, 使电解液达到氧饱和状态。

H 型电解池(H-Cell)测试 将待测样品分散液 (制备方法同上)均匀滴在碳纤维纸上, 负载量为 $1 \mathrm{mg} / \mathrm{cm}^{2}$ 。电解液为 $0.1 \mathrm{~mol} / \mathrm{L} \mathrm{KOH}$ 水溶液, 参比电 极为 $\mathrm{Ag} / \mathrm{AgCl}$ 电极, 对电极为碳棒, 电解池的隔膜 为 Nafion 211 膜。电化学测试前, 向电解液中持续 通入高纯氧气 $30 \mathrm{~min}$, 使电解液达到氧饱和状态。

电解液中双氧水浓度测试 使用硫酸钛比色法 测算双氧水浓度 ${ }^{[28]}$ 。其原理是双氧水与硫酸钛在强 酸性环境下反应生成过氧化物-钛复合物黄色溶液, 通过分光光度计检测其吸光度, 吸光度数值与过氧 化氢浓度在一定范围内呈线性关系, 结合外标法即 可计算电解液中双氧水浓度。具体来说, 先配置一 定浓度梯度的过氧化氢标准溶液, 将 $1 \mathrm{~mL}$ 标准溶
液与 $9 \mathrm{~mL}$ 硫酸钛溶液 $(1.4 \mathrm{~g}$ 硫酸钛与 $20 \mathrm{~mL}$ 浓硫酸 混合，用去离子水定容至 $100 \mathrm{~mL})$ 混合，充分反应 后进行紫外-可见光吸收光谱测试, 读取 $408 \mathrm{~nm}$ 波 长处的吸光度，对浓度绘制标准曲线。对于双氧水 浓度未知的电解液, 按相同的方法与硫酸钛溶液混 合反应后进行紫外 - 可见光吸收光谱测试，将 $408 \mathrm{~nm}$ 波长处的吸光度数值代入标准曲线的方程, 可算出电解液中双氧水浓度 ${ }^{[29]}$ 。

\section{2 结果与讨论}

\section{1 催化剂合成及表征}

用扫描电子显微镜 (SEM) 和透射电子显微镜 (TEM)观察材料的微观形貌(图 2)。从结果可以看出, 高缺陷石墨烯与低缺陷石墨烯在形貌上有较大区 别。低缺陷石墨烯(图 2(a))的尺寸较小，表面较为平 整，而高缺陷石墨烯(图 2(b))表面存在较多褶皱，石
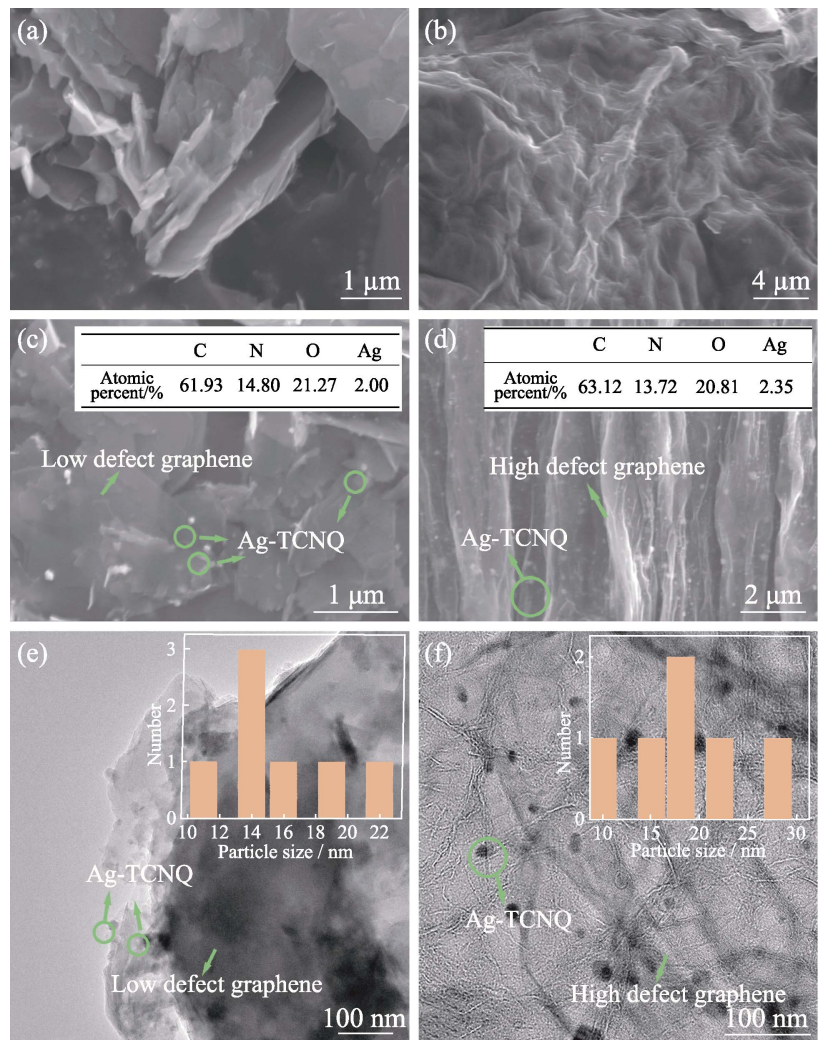

图 2 (a)低缺陷石墨烯和(b)高缺陷石墨烯的 SEM 照片, (c)Ag-TCNQ/低缺陷石墨烯复合材料和(d)Ag-TCNQ/高缺陷 石墨烯复合材料的 SEM 照片及元素含量分析(原子百分比); (e)Ag-TCNQ/低缺陷石墨烯复合材料和(f)Ag-TCNQ/高缺陷石 墨烯复合材料的 TEM 照片及粒径分布图

Fig. 2 SEM images of (a) low defect graphene, (b) high defect graphene, (c) Ag-TCNQ/low defect graphene, and (d) Ag- TCNQ/ high defect graphene with insets in (c, d) showing corresponding element analyses, TEM images and size distributions of (e) Ag$\mathrm{TCNQ} /$ low defect graphene and (f) Ag-TCNQ/high defect graphene 
墨烯片的尺寸较大。负载 Ag-TCNQ 之后, 两种材料 中(图 2(c, d))石墨烯本身的形貌并没有明显变化, 但是表面出现了纳米颗粒, 颗粒的尺寸接近, 元素 分析结果(图 2(c,d)插图)显示两种材料的 C、N、O、 $\mathrm{Ag}$ 含量基本相同。此外, 从透射电子显微镜(TEM) 照片中可以更清晰地观察到两种材料上负载的颗粒, 其直径都在 15 20 nm 之间(图 1(e,f))。

$X$ 射线衍射 (XRD) 可以确定产物的晶体结构。 如图(3(a,b))所示, 高缺陷石墨烯负载 Ag-TCNQ 后 出现了属于 Ag-TCNQ 的特征峰 $\left(2 \theta=10.4^{\circ}, 14.6^{\circ}\right.$, $\left.16.6^{\circ}, 20.8^{\circ}, 23.2^{\circ}, 26.9^{\circ}, 31.5^{\circ}\right)^{[30]}$, 说明 Ag-TCNQ 成功负载在高缺陷石墨烯表面。由于低缺陷石墨烯 片的晶体结构规整, 因此在 $2 \theta=27^{\circ}$ 左右出现了较高 强度的(002)特征峰, 导致其它特征峰显示不明显。 将谱图局部放大与 Ag-TCNQ 进行对比(图 3(b)插 图), 发现峰位基本吻合, 说明 Ag-TCNQ 也成功负 载在低缺陷石墨烯的表面。

石墨烯是一种零带隙的二维材料, 可通过原子 掺杂、化学修饰等方法打开石墨烯的带隙 ${ }^{[1-33]}$, 从 而提升其电化学性能。拉曼光谱中, $\mathrm{D}$ 峰 $\left(1350 \mathrm{~cm}^{-1}\right)$ 与 $\mathrm{G}$ 峰 $\left(1580 \mathrm{~cm}^{-1}\right)$ 的强度比 $\left(I_{\mathrm{D}} / I_{\mathrm{G}}\right)$ 可以表征石墨烯
中缺陷密度 ${ }^{[34]}$ 。如图 3(c, d) 所示, Ag-TCNQ/高缺陷 石墨烯、 $\mathrm{Ag}-\mathrm{TCNQ} /$ 低缺陷石墨烯、高缺陷石墨烯、 低缺陷石墨烯的 $I_{\mathrm{D}} / I_{\mathrm{G}}$ 比值分别为 $1.09,0.56,0.99$, 0.42 , 即材料的缺陷密度的关系为 $\mathrm{Ag}-\mathrm{TCNQ} /$ 高缺陷 石墨烯>高缺陷石墨烯>Ag-TCNQ/低缺陷石墨烯> 低缺陷石墨烯。这说明高缺陷石墨烯的缺陷密度高 于低缺陷石墨烯, 并且 Ag-TCNQ 与材料的复合会 增大材料的缺陷密度。

\section{2 电化学性能}

在旋转环盘电极(RRDE)装置测试中, 两种材料 的循环伏安 (CV)曲线(图 4(a)) 都在 $0.6 \mathrm{~V}$ (vs. RHE) 附近出现还原峰, 表明发生了氧还原反应。图 4(b) 为用 RRDE 测试得到的各种材料的线性扫描伏安 (LSV)曲线, 从结果可以看出, Ag-TCNQ、高缺陷石 墨烯、低缺陷石墨烯的起始电位都比 Ag-TCNQ/高 (低)缺陷石墨烯复合材料低; 同时, 相同电压下的电 流密度也明显低于 $\mathrm{Ag}-\mathrm{TCNQ} /$ 高(低)缺陷石墨烯复 合材料。这是因为石墨烯材料提供了高比表面积和 多孔结构, 非常有利于 ORR 的传质过程。而 AgTCNQ 纳米颗粒可以提供丰富的活性位点, 促进 ORR 过程最终形成复合效应, 提高了总体催化性能。
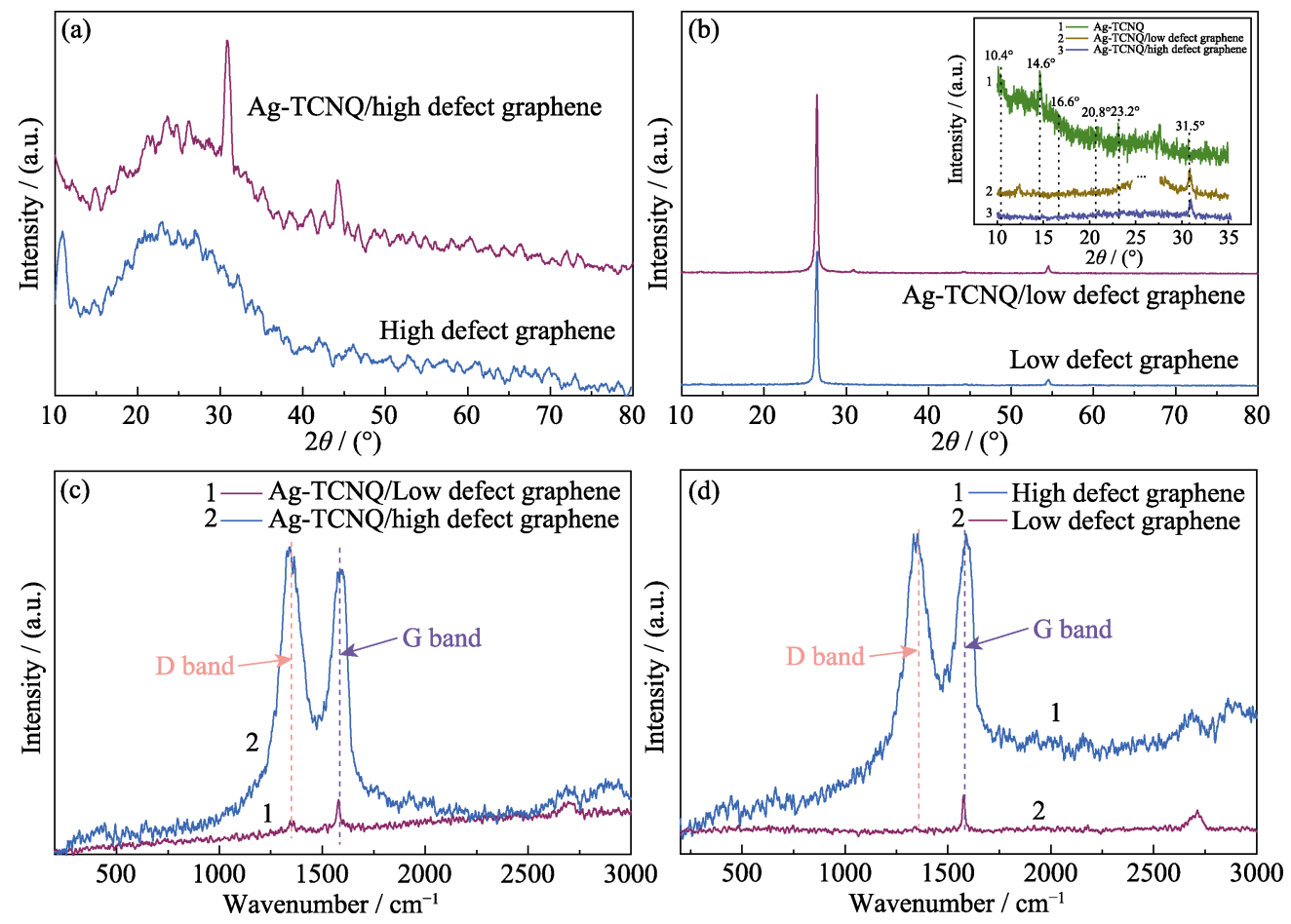

图 3 (a)高缺陷石墨烯、Ag-TCNQ/高缺陷石墨烯、(b)低缺陷石墨烯和 Ag-TCNQ/低缺陷石墨烯的 XRD 谱图, (b)插图为 Ag-TCNQ、Ag-TCNQ/高缺陷石墨烯及 Ag-TCNQ/低缺陷石墨烯的 XRD 局部放大图谱;

(c)Ag-TCNQ/低缺陷石墨烯和 Ag-TCNQ/高缺陷石墨烯, (d)低缺陷石墨烯和高缺陷石墨烯的拉曼光谱

Fig. 3 XRD patterns of (a) high defect graphene, Ag-TCNQ/high defect graphene, (b) low defect graphene and Ag-TCNQ/ low defect graphene with inset in (b) showing enlarged XRD profiles of Ag-TCNQ, Ag-TCNQ/high defect graphene and Ag-TCNQ low defect graphene; Raman spectra of (c) Ag- TCNQ/low defect graphene, Ag-TCNQ/high defect graphene, and (d) low defect graphene and high defect graphene 

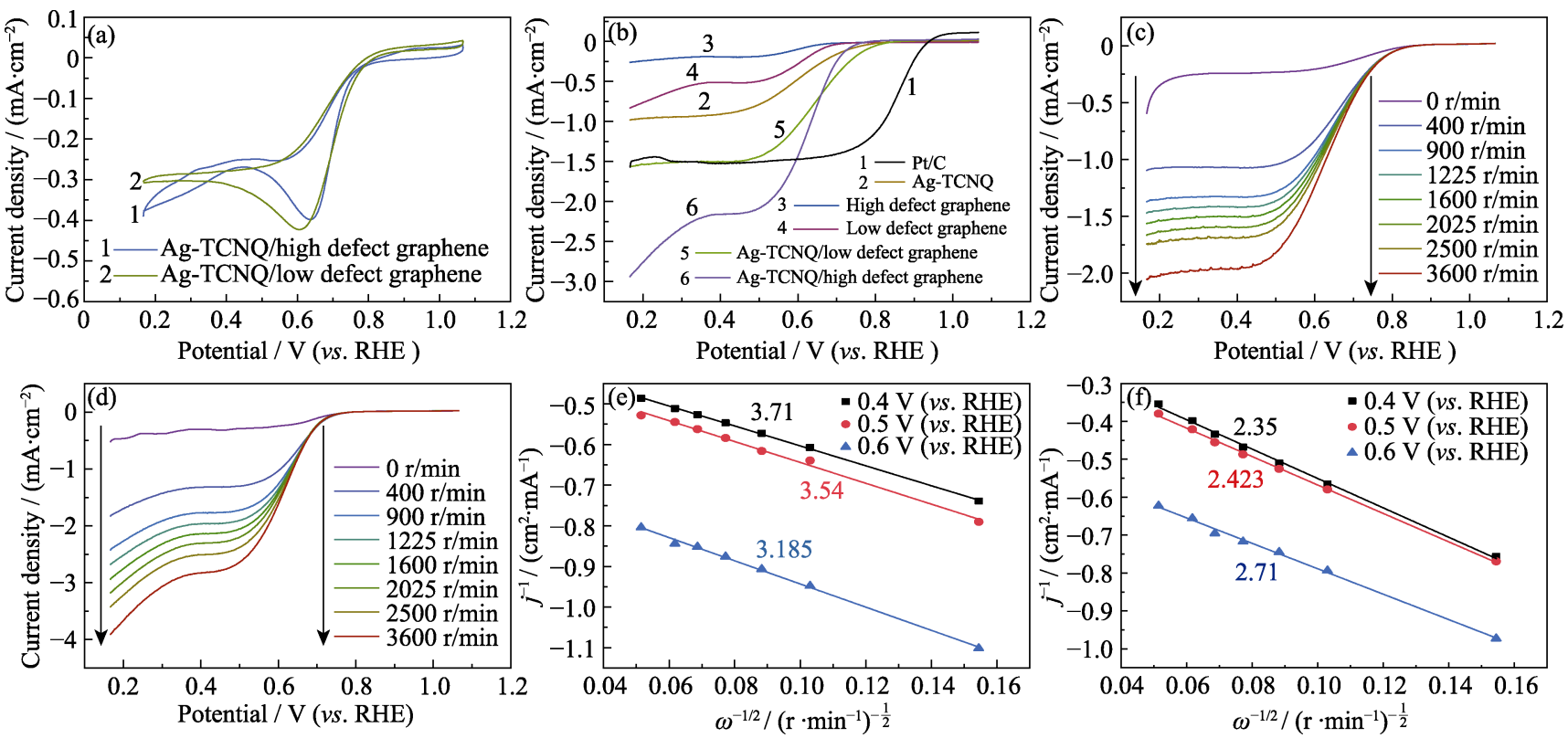

图 4 (a)Ag-TCNQ/低缺陷石墨烯和 Ag-TCNQ/高缺陷石墨烯的 $\mathrm{CV}$ 曲线; (b)Pt/C、Ag-TCNQ、高缺陷石墨烯、低缺陷石墨烯、 $\mathrm{Ag}-\mathrm{TCNQ} /$ 低缺陷石墨烯及 Ag-TCNQ/高缺陷石墨烯经 RRDE 测试得到的 LSV 曲线(转速为 $1600 \mathrm{r} / \mathrm{min}$ );

(c)Ag-TCNQ/低缺陷石墨烯和(d)Ag-TCNQ/高缺陷石墨烯在 RRDE 测试不同转速的 LSV 曲线;

(e)Ag-TCNQ/低缺陷石墨烯和; (f)Ag-TCNQ/高缺陷石墨烯的 K-L 曲线及电子转移数

Fig. 4 (a) CV curves of Ag-TCNQ/high defect graphene and Ag-TCNQ/low defect graphene; (b) LSV curves of Pt/C, Ag-TCNQ, low defect graphene, high defect graphene, Ag-TCNQ/low defect graphene and Ag-TCNQ/high defect graphene on RRDE at $1600 \mathrm{r} / \mathrm{min}$; LSV curves of (c) Ag-TCNQ/low defect graphene and (d) Ag-TCNQ/high defect graphene on RRDE at different rotating speeds in RRDE; K-L curves and electron transfer numbers calculated by K-L method of (e) Ag-TCNQ/low defect graphene and (f) Ag-TCNQ/high defect graphene

ORR 的电子转移数可以通过 K-L 法计算。氧还 原反应中电流的关系可用式(4)表示:

$$
\frac{1}{j}=\frac{1}{j_{\mathrm{K}}}+\frac{1}{j_{\mathrm{L}}}=\frac{1}{j_{\mathrm{K}}}+\frac{1}{B} \omega^{-1 / 2}
$$

其中, $j$ 为实验测得的电流 $(\mathrm{mA}) 、 j_{\mathrm{K}}$ 为动力学控制电 流 $(\mathrm{mA})$, 在同一电位下一般为常数, $j_{\mathrm{L}}$ 为传质控制 电流 $(\mathrm{mA})$, 与圆盘电极角速度 $\omega(\mathrm{r} / \mathrm{min})$ 的平方根成 比例，比例系数 $B$ 可用式(5)表示:

$$
B=0.62 D^{2 / 3} v^{-1 / 6} n F C^{*}
$$

其中, $D$ 为反应物的扩散系数 $\left(\mathrm{cm}^{2} / \mathrm{s}\right), v$ 为电解液的 动力学粘度 $\left(\mathrm{cm}^{2} / \mathrm{s}\right), F$ 为法拉第常数 $(96485 \mathrm{C} / \mathrm{mol})$, $C^{*}$ 为电解液中溶解氧的浓度 $\left(\mathrm{mol} / \mathrm{cm}^{3}\right)$ 。电子转移数 $n$ 可由 $j^{-1}$ 与 $\omega^{-1 / 2}$ 拟合直线的斜率( $\mathrm{K}-\mathrm{L}$ 斜率)求得。

结合线性扫描伏安曲线(图 4(c, d)), 可通过 K-L 法计算材料的电子转移数 (图 4(e, f)), 得到 Ag$\mathrm{TCNQ} /$ 高缺陷石墨烯复合材料的电子转移数为 2.4 , 接近二电子反应, 而 $\mathrm{Ag}-\mathrm{TCNQ} /$ 低缺陷石墨烯的电子 转移数为 3.7 , 接近四电子反应。可见, 在电催化反 应过程中, 增加石墨烯表面缺陷能促进二电子 ORR 过程, 而减少缺陷有利于四电子 $\mathrm{ORR}$ 过程的进行。

$\mathrm{Ag}-\mathrm{TCNQ} /$ 高缺陷石墨烯(测试电压 $0.5 \mathrm{~V}$ ( $v s$. RHE))和 Ag-TCNQ/低缺陷石墨烯(测试电压 $0.4 \mathrm{~V}$ (vs. RHE)) 的稳定性测试如图 5(a, b) 所示。 $24 \mathrm{~h}$ 的计 时电流测试中，电流密度基本稳定，并且测试前后 的 LSV 曲线(图 5(a, b)插图)几乎重合, 说明材料具 有较好的电化学稳定性。

本实验通过硫酸钛比色法测算电解液中双氧水 的含量, 标准曲线如图 5(c)所示。测试了两种材料 在 5 个不同电位下的双氧水产率(图 5(d)), Ag$\mathrm{TCNQ}$ /高缺陷石墨烯在电位为 $0.5 \mathrm{~V}$ ( $v s$. RHE) 时双 氧水产率达到最高 $(0.62 \mathrm{mg} / \mathrm{h})$, 此时法拉第效率为 $64.45 \%$ 。而对于 Ag-TCNQ/低缺陷石墨烯, 双氧水产 率始终很低, 并且随着电势增大, 产率进一步降低。

在 $\mathrm{Ag}-\mathrm{TCNQ}$ /石墨烯复合材料中, $\mathrm{Ag}-\mathrm{TCNQ}$ 纳 米颗粒及石墨烯相互作用, 产生了复合催化效应。石 墨烯的二维片层结构为 ORR 反应提供了良好的传质 条件，同时以石墨烯为基底，可显著抑制 Ag-TCNQ 纳米颗粒团聚，增大催化活性面积。通过计算双电层 电容比较催化剂材料电化学面积的大小, 在 0.87 $1.06 \mathrm{~V}$ (vs. RHE) 电势范围内测试两种材料不同扫速 下的 CV 曲线(图 6(a, c)), 选择电势为 $0.98 \mathrm{~V}$ (vs. RHE) 时的电流密度值, 取差值后除以 2 再与扫速作图(图 $6(b, d))$, 拟合得到的直线斜率即为材料的双电层电 容 $\left(C_{\mathrm{dl}}\right)$ 。通过计算可知, $\mathrm{Ag}-\mathrm{TCNQ} /$ 低缺陷石墨烯和 

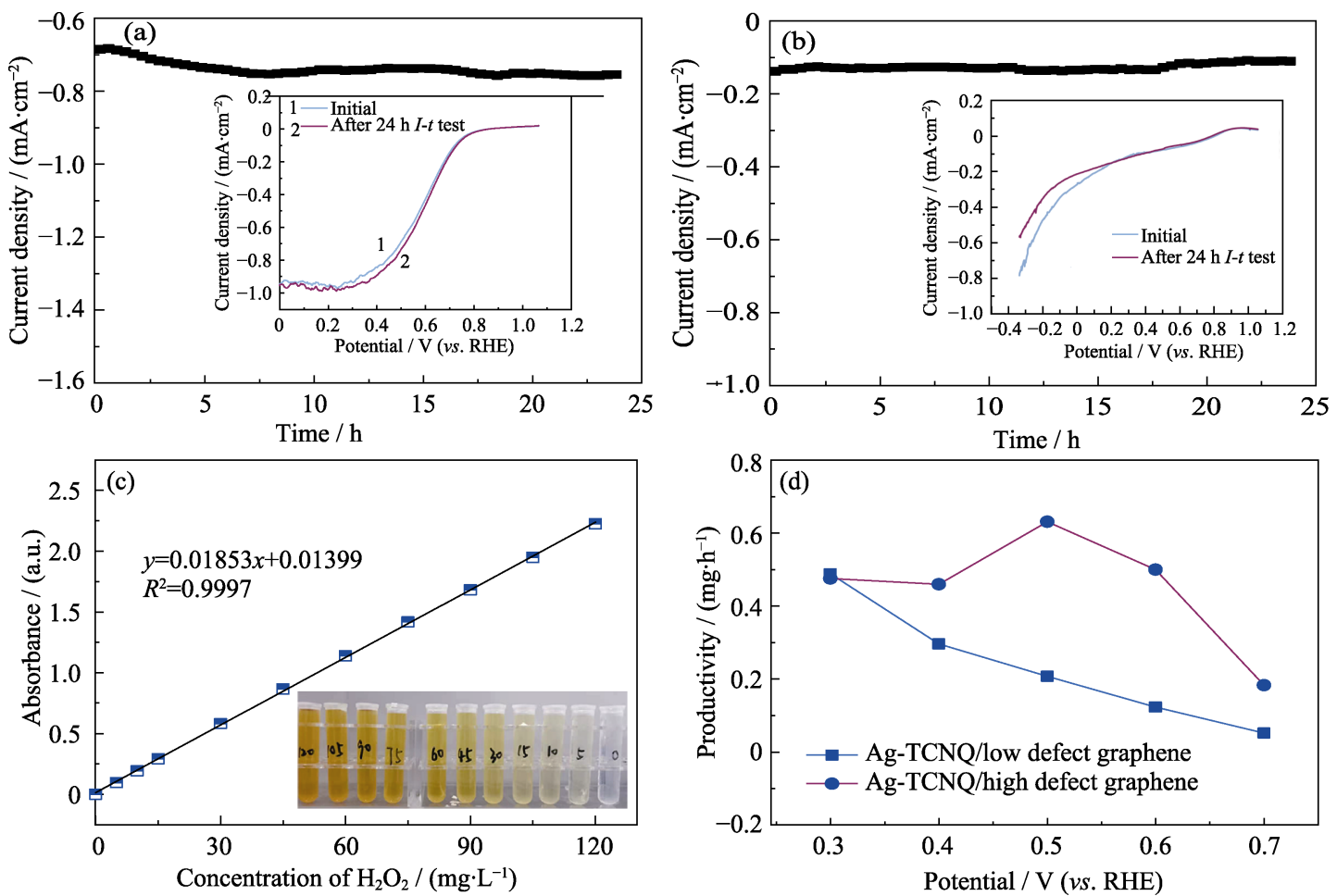

图 5 (a)Ag-TCNQ/高缺陷石墨烯, (b)Ag-TCNQ/低缺陷石墨烯的计时电流曲线, 插图为测试前后对应的 LSV 曲线; (c)双氧水浓度与吸光度的标准曲线; (d)Ag-TCNQ/高缺陷石墨烯和 Ag-TCNQ/低缺陷石墨烯在不同电位下的双氧水产率

Fig. 5 I- $t$ curves of (a) Ag-TCNQ/high defect graphene and (b) Ag-TCNQ/low defect graphene with insets showing corresponding LSV curves before and after test, (c) standard line of hydrogen peroxide concentration and UV-Vis absorbance , and

(d) production rates of Ag-TCNQ/high defect graphene and Ag-TCNQ/ low defect graphene at different potentials
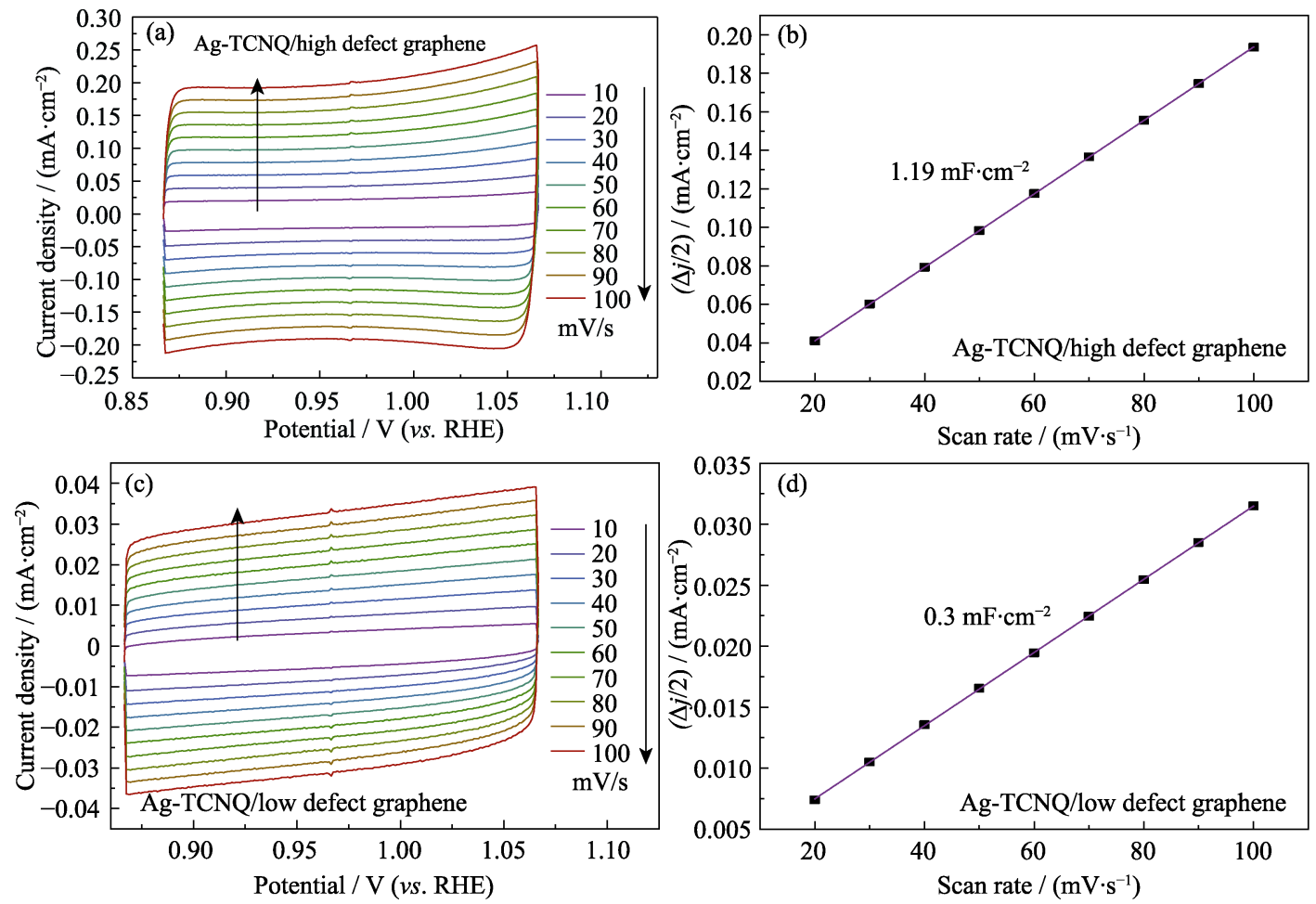

图 6 (a,b)Ag-TCNQ/高缺陷石墨烯和 $(\mathrm{c}, \mathrm{d}) \mathrm{Ag}-\mathrm{TCNQ} /$ 低缺陷石墨烯(a,c)不同扫速的 CV 曲线 $(0.87 \sim 1.06 \mathrm{~V}(v s . \mathrm{RHE}))$ 和 $(\mathrm{b}, \mathrm{d}) \Delta j / 2$ (电位为 $0.98 \mathrm{~V}(v s . \mathrm{RHE})$ )与扫描速率的函数关系

Fig. 6 (a,c) CV curves (0.87-1.06 V (vs. RHE)) at different scan rates and (b, d) corresponding capacitive $\Delta j / 2$ at $0.98 \mathrm{~V}(v s$. RHE) as a function of the scan rate for $(\mathrm{a}, \mathrm{b}) \mathrm{Ag}-\mathrm{TCNQ} /$ high defect graphene and $(\mathrm{c}, \mathrm{d}) \mathrm{Ag}-\mathrm{TCNQ} / \mathrm{low}$ defect graphene 
$\mathrm{Ag}-\mathrm{TCNQ} /$ 高缺陷石墨烯的双电层电容分别为 0.3 及 $1.91 \mathrm{mF} / \mathrm{cm}^{2}$, 说明 $\mathrm{Ag}-\mathrm{TCNQ} /$ 高缺陷石墨烯的电化 学活性面积更大, 这是因为石墨烯的表面缺陷改变 了其化学结构。

本研究所制备的 $\mathrm{Ag}-\mathrm{TCNQ} /$ 低缺陷石墨烯和 $\mathrm{Ag}-\mathrm{TCNQ} /$ 高缺陷石墨烯是用于电化学氧还原反应 的催化剂, 两种材料中唯一变量是石墨烯表面缺陷 的含量，却催化了两种不同的氧还原反应过程(即 二电子过程和四电子过程)。其中, $\mathrm{Ag}-\mathrm{TCNQ} /$ 低缺陷 石墨烯复合催化剂能促进 ORR 的四电子转移过程, 而 Ag-TCNQ/高缺陷石墨烯更有利于 ORR 的二电子 转移过程, 即缺陷更利于氧还原反应的二电子过程。

图 7 为反应机理示意图。一般来说, 导致 ORR 性能差异的原因是氧气在催化剂表面的吸附活化方 式不同的可能性最大 ${ }^{[35]}$ 。本研究采用 Hummers 法 制备高缺陷石墨烯，通过氧化打开石墨的层间距， 在石墨烯表面产生了丰富的含氧基团 ${ }^{[27]}$ 。这些含氧 基团成为石墨烯材料的结构缺陷，进而改变了石墨 烯表面的电子结构。由于含氧基团缺陷带有负电性, 促使氧气在石墨烯表面以端基式吸附活化，这种吸 附活化方式更有利于 ORR 二电子反应过程。而当缺 陷含量降低时，氧气在低缺陷石墨烯复合材料表面 的吸附方式偏向桥式吸附(如图 7 右侧), 更有利于 氧气的四电子还原过程。

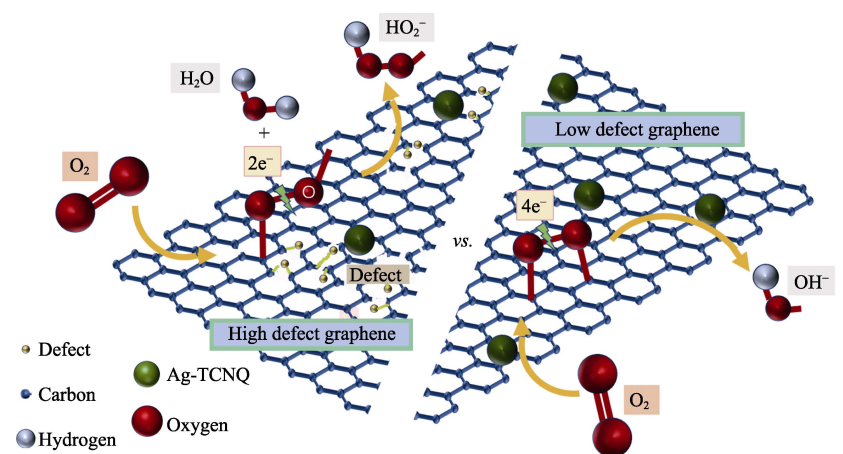

图 $7 \mathrm{Ag}-\mathrm{TCNQ}$ /高缺陷石墨烯(左)和 $\mathrm{Ag}-\mathrm{TCNQ} /$ 低缺陷石墨 烯(右)的表面氧还原反应示意图

Fig. 7 Schematic diagram of ORR on the surfaces of AgTCNQ/high defect graphene (left) and Ag-TCNQ/low defect graphene (right)

\section{3 结论}

将表面具有不同缺陷含量的石墨烯材料与 AgTCNQ 进行复合, 并测试和表征其结构及性能, 主 要结论如下:

1) 催化剂表面缺陷对 ORR 反应过程具有重要 的影响, 缺陷利于 ORR 进行二电子反应, 而低缺陷
的催化剂更倾向于促进四电子氧还原过程;

2) Ag-TCNQ 纳米颗粒为催化反应提供了更多 活性位点，而石墨烯作为载体可以抑制纳米颗粒团 聚，同时增大电化学活性面积。

从晶体学的角度来说，材料中不均匀的成分都 可以看作结构缺陷。缺陷根据不同的几何形态可分 为零维缺陷、一维缺陷、二维缺陷及三维缺陷等。 它们不但可以作为针点稳定金属或非金属原子(或 原子团簇), 也可以调控材料的电子结构, 从而改变 材料的性能。调控缺陷的类型(例如空位、掺杂等)、 增强缺陷的稳定性都是可供参考的优化催化剂性能 的方法。若能从根本上理解缺陷在催化中的作用及 机理，将会推动电催化领域进一步发展。

\section{参考文献:}

[1] BARACK O. The irreversible momentum of clean energy. Science, 2017, 355: 126-131.

[2] CHU S, MAJUMDAR A. Opportunities and challenges for a sustainable energy future. Nature, 2012, 488(7411): 294-303.

[3] ZHOU R, ZHENG Y, JARONIEC M, et al. Determination of the electron transfer number for the oxygen reduction reaction: from theory to experiment. ACS Catalysis, 2016, 6(7): 4720-4728.

[4] ZHOU W, RAJIC L, CHEN L, et al. Activated carbon as effective cathode material in iron-free electro-Fenton process: integrated $\mathrm{H}_{2} \mathrm{O}_{2}$ electrogeneration, activation, and pollutants adsorption. Electrochim Acta, 2019, 296: 317-326.

[5] SUN Y, SILVIOLI L, SAHRAIE N R, et al. Activity-selectivity trends in the electrochemical production of hydrogen peroxide over single-site metal-nitrogen-carbon catalysts. Journal of the American Chemical Society, 2019, 141(31): 12372-12381.

[6] WANG C, ZHAO H, WANG J, et al. Atomic Fe hetero-layered coordination between $\mathrm{g}_{-} \mathrm{C}_{3} \mathrm{~N}_{4}$ and graphene nanomeshes enhances the ORR electrocatalytic performance of zinc-air batteries. Journal of Materials Chemistry A, 2019, 7(4): 1451-1458.

[7] WANG J, KONG $\mathrm{H}$, ZHANG J, et al. Carbon-based electrocatalysts for sustainable energy applications. Progress in Materials Science, 2021, 116(17): 100717-100754.

[8] YANG L, SHUI J, DU L, et al. Carbon-based metal-free ORR electrocatalysts for fuel cells: past, present, and future. Advanced Materials, 2019, 31(13): e1804799.

[9] LI Y, LU J. Metal-air batteries: will they be the future electrochemical energy storage device of choice. ACS Energy Letters, 2017, 2(6): 1370-1377.

[10] ZHOU M, WANG H L, GUO S. Towards high-efficiency nanoelectrocatalysts for oxygen reduction through engineering advanced carbon nanomaterials. Chemical Society Reviews, 2016, 45(5): 1273-1307.

[11] YANG S, VERDAGUER-CASADEVALL A, ARNARSON L, et al. Toward the decentralized electrochemical production of $\mathrm{H}_{2} \mathrm{O}_{2}$ : a focus on the catalysis. ACS Catalysis, 2018, 8(5): 4064-4081.

[12] QIANG Z, CHANG J H, HUANG C P. Electrochemical generation of hydrogen peroxide from dissolved oxygen in acidic solutions. Water Research, 2002, 36: 85-95.

[13] CAMPOS-MARTIN J M, BLANCO-BRIEVA G, FIERRO J L G, Hydrogen peroxide synthesis: an outlook beyond the anthraquinone 
process. Angewandte Chemie International Edtion, 2006, 45(42): 6962-6984.

[14] EDWARDS J K, FREAKLEY S J, LEWIS R J, et al. Advances in the direct synthesis of hydrogen peroxide from hydrogen and oxygen. Catalysis Today, 2015, 248(7): 3-9.

[15] RUSSO V, TESSER R, SANTACESARIA E, et al. Chemical and technical aspects of propene oxide production via hydrogen peroxide (HPPO process). Industrial \& Engineering Chemistry Research, 2013, 52(3): 1168-1178.

[16] LUO M, YANG Y, GUO S. Precious metal nanocrystals for renewable energy electrocatalysis: structural design and controlled synthesis. Dalton Transaction, 2020, 49(2): 267-273.

[17] ASEFA T. Metal-free and noble metal-free heteroatom-doped nanostructured carbons as prospective sustainable electrocatalysts. Accounts of Chemical Research, 2016, 49(9): 1873-1883.

[18] CHEN Y, JI S, CHEN C, et al. Single-atom catalysts: synthetic strategies and electrochemical applications. Joule, 2018, 2(7): $1242-1264$.

[19] ZHU Y P, GUO C, ZHENG Y, et al. Surface and interface engineering of noble-metal-free electrocatalysts for efficient energy conversion processes. Accounts of Chemical research, 2017, 50(4): 915-923.

[20] TANG C, WANG H F, CHEN X, et al. Topological defects in metal-free nanocarbon for oxygen electrocatalysis. Advanced Materials, 2016, 28(32): 6845-6851.

[21] JIA Y, CHEN J, YAO X. Defect electrocatalytic mechanism: concept, topological structure and perspective. Materials Chemistry Frontiers, 2018, 2(7): 1250-1268.

[22] YAN X, JIA Y, YAO X. Defects on carbons for electrocatalytic oxygen reduction. Chemical Society Reviews, 2018, 47(20): $7628-7658$.

[23] DORRI MOGHADAM A, OMRANI E, MENEZES P L, et al. Mechanical and tribological properties of self-lubricating metal matrix nanocomposites reinforced by carbon nanotubes (CNTs) and graphene-a review. Composites Part B:Engineering, 2015, 77: $402-420$.

[24] GEIM A K. Graphene: status and prospects. Science, 2009,
324(19): 1530-1536.

[25] STOLLER M D, PARK S, ZHU Y, et al. Graphene-based ultracapacitors. Nano Letters, 2008, 8(10): 3498-3402.

[26] KUILLA T, BHADRA S, YAO D, et al. Recent advances in graphene based polymer composites. Progress in Polymer Science, 2010, 35(11): 1350-1375.

[27] KOTSYUBYNSKY V O, BOYCHUK V M, BUDZULIAK I M, et al. Structural properties of graphene oxide materials synthesized accordingly to Hummers, Tour and modified methods: XRD and Raman study. Physics and Chemistry of Solid State, 2021, 22(1): 31-38.

[28] MASTSUBARA C, KAWAMOTO N, TAKAMURA K. Oxo [5,10,15,20-tetra(4-pyridyl)porp hyrinatoltitanium (IV): an ultrahigh sensitivity spectrophotometric reagent for hydrogen peroxide. Analyst, 1992, 117: 1781-1784.

[29] EISENBERG G. Colorimetric determination of hydrogen peroxide. Industrial \& Engineering Chemistry, 1943, 15(5): 327-328.

[30] O'KANE S A, CLÉRAC R, ZHAO H, et al. New crystalline polymers of $\mathrm{Ag}(\mathrm{TCNQ})$ and $\mathrm{Ag}\left(\mathrm{TCNQF}_{4}\right)$ : structures and magnetic properties. Journal of Solid State Chemistry, 2000, 152(1): 159-173.

[31] ZHANG Q, ZHANG X, WANG J, et al. Graphene-supported single-atom catalysts and applications in electrocatalysis. Nanotechnology, 2021, 32(3): 1-24.

[32] MOHAN V B, LAU K T, HUI D, et al. Graphene-based materials and their composites: a review on production, applications and product limitations. Composites Part B: Engineering, 2018, 142: 200-220.

[33] HAN L, SUN Y, LI S, et al. In-plane carbon lattice-defect regulating electrochemical oxygen reduction to hydrogen peroxide production over nitrogen-doped graphene. ACS Catalysis, 2019, 9(2): 1283-1288.

[34] MALARD L M, PIMENTA M A, DRESSElhaUS G, et al. Raman spectroscopy in graphene. Physics Reports, 2009, 473(5/6): $51-87$.

[35] JIRKOVSKY J S, PANAS I, AHLBERG E, et al. Single atom hot-spots at Au-Pd nanoalloys for electrocatalytic $\mathrm{H}_{2} \mathrm{O}_{2}$ production. Journal of the American Chemical Society, 2011, 133(48): 19432-19441. 